\title{
Multiple Myeloma-Light Chain Only
}

National Cancer Institute

\section{Source}

National Cancer Institute. Multiple Myeloma-Light Chain Only. NCI Thesaurus. Code C70605.

Only the light chain (kappa or lambda) of the immunoglobulin is defective. 